\title{
Elevated glucose level leads to rapid COVID-19 progression and high fatality
}

Wenjun Wang ${ }^{1,2,3 \dagger}{ }^{+}$, Mingwang Shen ${ }^{4 \dagger}$, Yusha Tao ${ }^{4}$, Christopher K. Fairley ${ }^{4,5}$, Qin Zhong ${ }^{1,2,3}$, Zongren Li ${ }^{1,2,3}$, Hui Chen ${ }^{6,7}$, Jason J. Ong ${ }^{4,5}$, Dawei Zhang ${ }^{8}$, Kai Zhang ${ }^{1}$, Ning Xing ${ }^{10}$, Huayuan Guo ${ }^{1}$, Enqiang Qin ${ }^{8}$, Xizhou Guan ${ }^{11}$, Feifei Yang ${ }^{1}$, Sibing Zhang ${ }^{6,9}$, Lei Zhang ${ }^{4,5,12^{*}}$ and Kunlun $\mathrm{He}^{1,2,3^{*}}$

\begin{abstract}
Objectives: We aimed to identify high-risk factors for disease progression and fatality for coronavirus disease 2019 (COVID-19) patients.

Methods: We enrolled 2433 COVID-19 patients and used LASSO regression and multivariable cause-specific Cox proportional hazard models to identify the risk factors for disease progression and fatality.

Results: The median time for progression from mild-to-moderate, moderate-to-severe, severe-to-critical, and criticalto-death were 3.0 (interquartile range: 1.8-5.5), 3.0 (1.0-7.0), 3.0 (1.0-8.0), and 6.5 (4.0-16.3) days, respectively. Among 1,758 mild or moderate patients at admission, 474 (27.0\%) progressed to a severe or critical stage. Age above 60 years, elevated levels of blood glucose, respiratory rate, fever, chest tightness, c-reaction protein, lactate dehydrogenase, direct bilirubin, and low albumin and lymphocyte count were significant risk factors for progression. Of 675 severe or critical patients at admission, 41 (6.1\%) died. Age above 74 years, elevated levels of blood glucose, fibrinogen and creatine kinase-MB, and low plateleta count were significant risk factors for fatality. Patients with elevated blood glucose level were 58\% more likely to progress and 3.22 times more likely to die of COVID-19.
\end{abstract}

Conclusions: Older age, elevated glucose level, and clinical indicators related to systemic inflammatory responses and multiple organ failures, predict both the disease progression and the fatality of COVID-19 patients.

Keywords: COVID-19, Progression, Fatality, Risk factors

\section{Introduction}

In December 2019, an outbreak of novel coronavirus pneumonia (COVID-19) caused by SARS-CoV-2 was reported in Wuhan city, China. Since then, COVID-19 had rapidly spread to more than 17.6 million cases, with

*Correspondence: lei.zhang1@xjtu.edu.cn; kunlunhe@plagh.org

tWenjun Wang and Mingwang Shen contributed equally to this work ${ }^{\dagger}$ Lei Zhang and Kunlun He supervised equally to this work

${ }^{1}$ Key Laboratory of Ministry of Industry and Information Technology of Biomedical Engineering and Translational Medicine, Chinese PLA

General Hospital, Beijing 100853, People's Republic of China

${ }^{4}$ China-Australia Joint Research Center for Infectious Diseases, School of Public Health, Xi'an Jiaotong University Health Science Center, Xi'an, Shanxi 710061, People's Republic of China

Full list of author information is available at the end of the article over 680,000 deaths worldwide as of August 2, 2020 [1] A total of 84,428 cases and 4,634 deaths were reported in China, and approximately $81 \%$ of cases and $97 \%$ of deaths were from Wuhan city of August 2, 2020 [2]. As the hardest-hit city by the COVID-19 pandemic, Wuhan initiated a metropolitan-wide quarantine on January 23, 2020, which terminated all public transportation in the city and intercity links. The quarantine lasted for 76 days and was eventually lifted on April 8, 2020 [3-6]. The metropolitan-wide quarantine approach had since become a common practice to combat the COVID-19 epidemic globally.

At the peak of the epidemic, Wuhan authorities constructed an emergency hospital specialized in treating

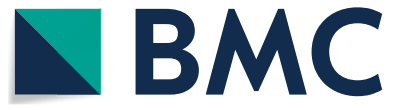

(c) The Author(s) 2021. Open Access This article is licensed under a Creative Commons Attribution 4.0 International License, which permits use, sharing, adaptation, distribution and reproduction in any medium or format, as long as you give appropriate credit to the original author(s) and the source, provide a link to the Creative Commons licence, and indicate if changes were made. The images or other third party material in this article are included in the article's Creative Commons licence, unless indicated otherwise in a credit line to the material. If material is not included in the article's Creative Commons licence and your intended use is not permitted by statutory regulation or exceeds the permitted use, you will need to obtain permission directly from the copyright holder. To view a copy of this licence, visit http://creativecommons.org/licenses/by/4.0/. The Creative Commons Public Domain Dedication waiver (http://creativeco mmons.org/publicdomain/zero/1.0/) applies to the data made available in this article, unless otherwise stated in a credit line to the data. 
people infected with SARS-CoV-2, inspired by the prefab hospital erected in Beijing during the 2003 SARS outbreak. This emergency hospital, Huoshenshan hospital, was constructed within ten days and the first hospital specialized in treating patients with COVID-19 in the world [7-9]. This hospital closed on April 15, 2020, one week after the metropolitan-wide quarantine was lifted [10]. During the 73 days of operation, the hospital treated 3,059 patients with COVID-19, of whom 2,961 recovered [7].

Identifying the risk factors of COVID-19 disease progression and fatality provides important evidence to support appropriate clinical management and optimize medical resource allocation [11-13]. The risk factors for COVID-19 progression include comorbidities with chronic diseases (hypertension, diabetes, cardiovascular disease and liver disease), old age, low lymphocyte and albumin counts and elevated levels of lactate dehydrogenase, c-reactive protein, red blood cell distribution width, blood urea nitrogen and direct bilirubin [14]. In particular, patients with old age, neutrophilia, thrombocytopenia, higher lactate dehydrogenase and d-dimer levels were more likely to develop Acute Respiratory Distress Syndrome (ARDS) [15, 16]. Further, older age, d-dimer level greater than $1 \mu \mathrm{g} / \mathrm{mL}$ and a high Sequential Organ Failure Assessment score on admission would contribute to a higher in-hospital fatality of COVID-19 patients [17]. Liang et al. [18], based on chest radiography abnormality and nine clinical indicators were able to predict the risk of developing critical illness with an area-underthe-curve of 0.88. A systematic review and meta-analysis examine risk factors associated with adverse clinical outcomes in patients with COVID-19 [19]. However, most of these studies were limited by their relatively small sample sizes, and many patients had not progressed to the study endpoints by the time the study was conducted, leading to bias and unreliable prediction for disease progression and fatality. Besides, some analyses of risk factors were not adjusted for potential confounding effects, leading to false associations.

In this study, we retrospectively collected the complete hospitalization information from 2,433 patients who were admitted to Huoshenshan hospital during its 73 days of operation. We explored on the time for disease progression among patients in various disease stages at admission and determined their risk of disease progression. We also identified clinical risk factors that predict the COVID-19 disease progression and fatality among these patients.

\section{Methods}

\section{Study design and patients}

We established a retrospective observational study cohort, based on 3,059 cases admitted to the Huoshenshan hospital in Wuhan between February 4 and April 15, 2020. The exclusion criteria were: (1) Patients who were not confirmed by a positive result of severe acute respiratory syndrome coronavirus 2 detection in respiratory specimens by the reverse transcriptase polymerase chain reaction assay, or in serum by the specific IgM and IgG antibody detection; (2) Patients who referred to other medical institution during hospitalization; (3) Patients who were admitted to the hospital multiple times; (4) Patients were younger than 18 years old; (5) Patients without laboratory data included in this study within the $24 \mathrm{~h}$ after admission. This study tracked the progression of COVID-19 patients from admission until one of the endpoints (discharged or death).

\section{Clinical and outcome indicators}

Demographic, clinical, laboratory, treatment, and clinical outcome data were obtained from the hospital's electronic clinical medical records. At the first clinical consultation, demographic, clinical and laboratory data were collected within the first day after admission. Treatment data and clinical outcomes (including the event of disease progression, time of each disease stages, fatality, duration of hospitalization and endpoint status) were also collected during the course from admission to the study endpoints. We defined the event of disease progression as a mild or moderate patient at admission would progress to severe or critical stage at the first time during hospitalization.

\section{Clinical definitions}

The severity of COVID-19 was defined according to the Guidance 7th edition [20]. Patients were classified as 'mild' if there was no evidence of pneumonia on imaging nor any of the features for moderate or higher severity; as 'moderate' if they had evidence of pneumonia on imaging but no features of severe or higher severity; as 'severe' if they meet any of the following criteria: (1) respiratory distress ( $\geq 30$ breaths/min); (2) oxygen saturation $\leq 93 \%$ at rest on room air; (3) arterial partial pressure of oxygen $(\mathrm{PaO} 2)$ or fraction of inspired oxygen $(\mathrm{FiO} 2) \leqq 300 \mathrm{mmHg}(\mathrm{mmHg}=0.133 \mathrm{kPa})$; and as 'critical' if they required mechanical ventilation, had a septic shock or required admission to ICU. Comorbidities were defined according to ICD10-CM code [21]. Detailed definitions for clinical symptoms were provided in the supplemental materials. We considered a patient progressing to a severe or critical disease stage when the individual had none of the severe or critical stages at admission 
but developed these stages for the first time during hospitalization.

Patients had to meet all the following criteria before being discharged: (1) body temperature returned to normal $\left(<37.5^{\circ} \mathrm{C}\right)$ for three consecutive days; (2) respiratory symptoms improved substantially; (3) pulmonary imaging showed an obvious absorption of inflammation; and (4) two consecutive negative nuclei acid tests, each at least $24 \mathrm{~h}$ apart.

\section{Statistical analysis}

We presented continuous variables as the median and interquartile range (IQR) and examined the differences between disease severity groups using the Kruskal-Wallis one-way ANOVA We presented categorical variables with the corresponding percentage and examined the differences using $\chi^{2}$ test or Fisher's exact test. We conducted survival analyses on disease progression and fatality based on a competing risk framework. The outcome variables included: (1) the event of the first time disease progression to severe or critical disease states among mild or moderate patients at admission, and (2) in-hospital fatality among patients with severe or critical at admission. Discharge from the hospital was considered as a competing risk event. Five clinical indicators (interleukin-6, natriuretic peptide type B, supersensitive troponin I, myoglobin and procalcitonin.) with more than $30 \%$ of entries missing were excluded from the analysis. Data imputation was performed if missing percentage $<30 \%$ using Multivariate Imputation by Chained Equations. Statistically significant variables in the univariate analysis were ranked and further selected using LASSO regression [22]. The number of variables was defined as the number of variables when $\lambda=\lambda$ min in LASSO, or the total number of events divided by 10 (event per variable $>10$ rule)[23], whichever is smaller. Variables, which particularly reported in previous literatures were included in final analysis. The pooled set of variables were then included for the final multivariable cause-specific Cox proportional hazard model. Cumulative incidence curves were plotted to demonstrate the incidence of differences between different risk levels of key variables. A $p$ value of $<0.05$ was considered statistically significant. Statistical analyses were conducted using the $\mathrm{R}$ software (version 3.6.1).

\section{Results}

\section{Demographic characteristics of patients}

After excluding 214 patients who were only diagnosed clinically according to the Guidance 7th edition [20], 46 patients who referred to other medical institution, 16 patients who were admitted to the hospital multiple times, six patients were younger than 18 years old.
Further, we excluded 328 patients without laboratory data included in this study within the $24 \mathrm{~h}$ after admission, we included 2,433 COVID-19 patients in the final analysis. Fifty patients died during hospitalization, and 2,383 were discharged, corresponding to a case-fatality ratio of $2.1 \%$. Patient's median age was 60.0 years (IQR $50.0-68.0$ ), and $50.2 \%$ were male (Table 1 ). The most common symptoms or signs on admission were cough (55.7\%), fatigue (38.9\%), and shortness of breath (25.0\%). Hypertension being the most common comorbidity $(31.6 \%)$, followed by diabetes $(14.3 \%)$ and coronary heart disease (6.7\%). During hospitalization, 847 (34.8\%) patients received antibiotics, $1233(50.7 \%)$ received antivirals, and $68(2.8 \%)$ received non-invasive mechanical ventilation and $42(1.7 \%)$ received invasive mechanical ventilation.

\section{Clinical progression and regression during hospitalization}

Among 25 mild patients at admission, 19 retained mild and were discharged after 6.0 (5.0-11.0) days; six patients who progressed to moderate severity in $3.0(1.8-5.5)$ days, but all discharged after another 8.0 (6.8-8.8) days. Of 1,733 moderate patients at admission, 1,259 patients retained moderate and discharged after 11.0 (7.0-16.0) days. In contrast, 474 patients progressed to the severe state in 3.0 (1.0-7.0) days, but all recovered and were discharged after another 12.0 (6.5-18.0) days, and 9 patients deceased after $9.0(2.5-19.0)$ days. Of 635 severe patients at admission, 604 patients regressed to moderate severity in $7.0(5.0-11.0)$ days and were discharged after another 6.0 (4.0-11.0) days. Thirty-one patients progressed to critical severity after $3.0(1.8-8.0)$ days, and of whom only 10 patients were discharged after 15.5 (14.0-30.5) days, and 21 died after $6.0(0.0-10.5)$ days. Of 40 critical patients at admission, 20 patients regressed to moderate severity after 10.5 (8.3-15.8) days and were discharged after another 11.5 (6.3-19.5) days, and the remaining 20 patients died after 6.5 (4.0-16.3) days (Figs. 1 and 2).

Across all patients, it required a median of $3.0(1.8-5.5)$ days to progress from mild to moderate, 3.0 (1.0-7.0) days from moderate to severe, $3.0(1.0-8.0)$ days from severe to critical and $6.5(4.0-16.3)$ from critical to fatality. In contrast, it required $7.0(5.0-11.0)$ days to regress from severe or critical to moderate severity and 6.5 (4.0-11.0) days from moderate to discharge. The median admission-to-discharge time among mild, moderate, severe and critical patients on admission were 7.0 (5.512.0), 13.0 (9.0-19.0), 15.0 (8.0-22.0), and 25.0 (15.334.8 ) days, respectively. 
Table 1 Basic demographic characteristics, signs and symptoms, comorbidities, laboratory findings, treatment and clinical outcomes of 2,433 COVID-19 patients admitted to the Huoshenshan hospital

\begin{tabular}{|c|c|c|c|c|c|c|}
\hline \multirow[t]{2}{*}{ Variable } & \multirow[t]{2}{*}{ All patients $(\mathrm{N}=2,433)$} & \multicolumn{4}{|c|}{ Clinical classification at admission } & \multirow[t]{2}{*}{$p$ value } \\
\hline & & Mild $(\mathrm{N}=25)$ & Moderate $(\mathrm{N}=1,733)$ & Severe $(N=635)$ & Critical $(\mathrm{N}=40)$ & \\
\hline \multicolumn{7}{|l|}{$\begin{array}{l}\text { Demographic charac- } \\
\text { teristics }\end{array}$} \\
\hline Age (IQR)-year & $60.0(50.0,68.0)$ & $36.0(29.0,54.0)$ & $58.0(48.0,66.0)$ & $65.0(56.0,72.0)$ & $67.0(58.0,80.3)$ & $<0.001^{* *}$ \\
\hline Male gender-no. (\%) & $1222(50.2)$ & $14(56.0)$ & $891(51.4)$ & $301(47.4)$ & $16(40.0)$ & 0.171 \\
\hline $\begin{array}{l}\text { Smoking history-no. } \\
(\%)\end{array}$ & $205(8.4)$ & $3(12.0)$ & $145(8.4)$ & $52(8.2)$ & $5(12.5)$ & 0.592 \\
\hline $\begin{array}{l}\text { Drinking history—no. } \\
(\%)\end{array}$ & $154(6.3)$ & $1(4.0)$ & $117(6.8)$ & $32(5.0)$ & $4(10.0)$ & 0.291 \\
\hline Respiratory rate > $20(\%)$ & $689(28.3)$ & $4(16.0)$ & $426(24.6)$ & $232(36.5)$ & $27(67.5)$ & $<0.001^{* *}$ \\
\hline Pulse rate > $100(\%)$ & $356(14.6)$ & $3(12.0)$ & $233(13.4)$ & $106(16.7)$ & $14(35.0)$ & $0.001^{*}$ \\
\hline $\begin{array}{l}\text { Systolic blood pres- } \\
\text { sure } \geq 140(\%)\end{array}$ & $655(26.9)$ & $6(24.0)$ & $433(25.0)$ & $203(32.0)$ & $13(32.5)$ & $0.007^{*}$ \\
\hline $\begin{array}{l}\text { Diastolic blood pres- } \\
\text { sure } \geq 90(\%)\end{array}$ & $562(23.1)$ & $3(12.0)$ & $414(23.9)$ & $139(21.9)$ & $6(15.0)$ & 0.227 \\
\hline \multicolumn{7}{|l|}{$\begin{array}{l}\text { Signs and symptoms- } \\
\text { no. (\%) }\end{array}$} \\
\hline $\begin{array}{l}\text { Body temperature } \\
\text { (IQR) - }{ }^{\circ} \mathrm{C}\end{array}$ & $36.5(36.3,36.7)$ & $36.5(36.3,36.7)$ & $36.5(36.3,36.7)$ & $36.5(36.3,36.7)$ & $36.5(36.3,36.8)$ & $0.022^{*}$ \\
\hline $\begin{array}{l}\text { Fever (tempera- } \\
\text { ture } \geq 37.5^{\circ} \mathrm{C} \text { ) }\end{array}$ & $63(2.6)$ & $0(0.0)$ & $34(2.0)$ & $27(4.3)$ & $2(5.0)$ & $0.012^{*}$ \\
\hline Cough & $1356(55.7)$ & $3(12.0)$ & $969(55.9)$ & $363(57.2)$ & $21(52.5)$ & $<0.001^{* *}$ \\
\hline Fatigue & $947(38.9)$ & $4(16.0)$ & $677(39.1)$ & $251(39.5)$ & $15(37.5)$ & 0.116 \\
\hline Diarrhea & $65(2.7)$ & $2(8.0)$ & $44(2.5)$ & $18(2.8)$ & $1(2.5)$ & 0.288 \\
\hline Chest tightness & $292(12.0)$ & $0(0.0)$ & $190(11.0)$ & $97(15.3)$ & $5(12.5)$ & $0.007^{*}$ \\
\hline Shortness of breath & $608(25.0)$ & $0(0.0)$ & $411(23.7)$ & $189(29.8)$ & $8(20.0)$ & $<0.001^{* *}$ \\
\hline \multicolumn{7}{|l|}{ Comorbidities-no. (\%) } \\
\hline Hypertension & 769 (31.6) & $5(20.0)$ & $497(28.7)$ & $252(39.7)$ & $15(37.5)$ & $<0.001^{* *}$ \\
\hline Diabetes & $349(14.3)$ & $0(0.0)$ & $233(13.4)$ & $109(17.2)$ & $7(17.5)$ & $0.013^{*}$ \\
\hline Coronary heart disease & $163(6.7)$ & $1(4.0)$ & $94(5.4)$ & $64(10.1)$ & $4(10.0)$ & $<0.001^{* *}$ \\
\hline Cancer & $42(1.7)$ & $0(0.0)$ & $29(1.7)$ & $12(1.9)$ & $1(2.5)$ & 0.739 \\
\hline Chronic bronchitis & $53(2.2)$ & $0(0.0)$ & $30(1.7)$ & $19(3.0)$ & $4(10.0)$ & $0.009^{*}$ \\
\hline Cerebrovascular disease & $89(3.7)$ & $0(0.0)$ & $48(2.8)$ & $37(5.8)$ & $4(10.0)$ & $<0.001^{* *}$ \\
\hline Chronic kidney disease & $43(1.8)$ & $0(0.0)$ & $23(1.3)$ & $17(2.7)$ & $3(7.5)$ & $0.011^{*}$ \\
\hline $\begin{array}{l}\text { Chronic obstructive } \\
\text { pulmonary disease }\end{array}$ & $19(0.8)$ & $0(0.0)$ & $9(0.5)$ & $9(1.4)$ & $1(2.5)$ & $0.059^{*}$ \\
\hline Hepatitis & $23(0.9)$ & $1(4.0)$ & $19(1.1)$ & $3(0.5)$ & $0(0.0)$ & 0.189 \\
\hline \multicolumn{7}{|l|}{ Laboratory findings (IQR) } \\
\hline C-reactive protein $(\mathrm{mg} / \mathrm{L})$ & $2.1(0.8,7.4)$ & $0.5(0.2,2.5)$ & $1.8(0.7,5.3)$ & $3.4(1.2,14.8)$ & $45.0(8.7,104.0)$ & $<0.001^{* *}$ \\
\hline D-dimer (mg/L) & $0.4(0.2,0.8)$ & $0.2(0.2,0.3)$ & $0.3(0.2,0.6)$ & $0.6(0.3,1.2)$ & $1.6(1.0,4.1)$ & $<0.001^{* *}$ \\
\hline $\begin{array}{l}\text { Lactate dehydrogenase } \\
(\mathrm{IU} / \mathrm{L})\end{array}$ & $175.0(150.3,211.7)$ & $150.7(128.9,181.3)$ & $169.1(147.2,199.7)$ & $193.8(163.7,241.4)$ & $332.3(250.7,430.9)$ & $0.002^{*}$ \\
\hline $\begin{array}{l}\text { White blood cell count } \\
\left(10^{9} / \mathrm{L}\right)\end{array}$ & $5.7(4.7,7.0)$ & $5.7(4.8,7.0)$ & $5.6(4.7,6.8)$ & $5.8(4.7,7.2)$ & $9.3(7.5,14.3)$ & $<0.001^{* *}$ \\
\hline $\begin{array}{l}\text { Lymphocyte count } \\
\left(10^{9} / \mathrm{L}\right)\end{array}$ & $1.5(1.1,1.9)$ & $1.7(1.5,2.0)$ & $1.5(1.2,1.9)$ & $1.4(0.9,1.7)$ & $0.8(0.5,1.3)$ & $<0.001^{* *}$ \\
\hline $\begin{array}{l}\text { Neutrophils count } \\
\left(10^{9} / \mathrm{L}\right)\end{array}$ & $3.5(2.7,4.6)$ & $3.4(2.5,4.2)$ & $3.4(2.6,4.4)$ & $3.7(2.8,5.1)$ & $8.4(5.6,13.5)$ & $<0.001^{* *}$ \\
\hline Monocyte count $\left(10^{9} / \mathrm{L}\right)$ & $0.4(0.3,0.5)$ & $0.4(0.3,0.5)$ & $0.4(0.3,0.5)$ & $0.4(0.3,0.6)$ & $0.4(0.2,0.6)$ & $<0.001^{* *}$ \\
\hline Basophils count $\left(10^{8} / \mathrm{L}\right)$ & $0.2(0.1,0.3)$ & $0.2(0.1,0.3)$ & $0.2(0.1,0.3)$ & $0.2(0.1,0.3)$ & $0.0(0.0,0.3)$ & $<0.001^{* *}$ \\
\hline Eosinophils count $\left(10^{8} / \mathrm{L}\right)$ & $1.1(0.6,1.8)$ & $1.3(0.9,1.8)$ & $1.1(0.6,1.8)$ & $1.1(0.5,1.9)$ & $0.2(0.0,1.1)$ & $<0.001^{* *}$ \\
\hline Prothrombin time (s) & $12.8(12.2,13.5)$ & $12.8(12.3,13.3)$ & $12.8(12.2,13.4)$ & $12.9(12.3,13.7)$ & $14.1(12.6,16.6)$ & 0.256 \\
\hline
\end{tabular}


Table 1 (continued)

\begin{tabular}{|c|c|c|c|c|c|c|}
\hline \multirow[t]{2}{*}{ Variable } & \multirow[t]{2}{*}{ All patients $(\mathrm{N}=2,433)$} & \multicolumn{4}{|c|}{ Clinical classification at admission } & \multirow[t]{2}{*}{$p$ value } \\
\hline & & Mild $(\mathrm{N}=25)$ & Moderate $(\mathrm{N}=1,733)$ & Severe $(N=635)$ & Critical $(N=40)$ & \\
\hline Total bilirubin ( $\mu \mathrm{mol} / \mathrm{L})$ & $9.6(7.4,12.5)$ & $9.5(7.3,10.9)$ & $9.5(7.3,12.4)$ & $9.8(7.7,12.6)$ & $11.3(8.4,16.1)$ & 0.561 \\
\hline Direct bilirubin $(\mu \mathrm{mol} / \mathrm{L})$ & $3.4(2.5,4.5)$ & $3.4(2.9,3.9)$ & $3.3(2.5,4.3)$ & $3.5(2.6,4.8)$ & $5.6(3.5,8.9)$ & $<0.001^{* *}$ \\
\hline Albumin (g/L) & $38.4(35.5,40.7)$ & $41.1(38.9,43.9)$ & $38.8(36.1,41.0)$ & $37.1(33.9,39.5)$ & $33.3(29.1,37.2)$ & $<0.001^{* *}$ \\
\hline $\begin{array}{l}\text { Alkaline phosphatase } \\
\text { (IU/L) }\end{array}$ & $69.7(58.3,84.5)$ & $66.6(57.7,78.1)$ & $68.6(57.7,83.8)$ & $71.7(59.7,84.7)$ & $92.9(77.7,114.3)$ & 0.202 \\
\hline Fibrinogen (g/L) & $3.0(2.6,3.4)$ & $2.5(2.4,3.1)$ & $3.0(2.6,3.3)$ & $3.0(2.7,3.5)$ & $3.0(2.6,3.8)$ & $0.007^{*}$ \\
\hline Creatinine $(\mu \mathrm{mol} / \mathrm{L})$ & $64.1(54.7,75.3)$ & $64.6(52.9,79.5)$ & $63.5(54.7,74.6)$ & $65.4(55.2,77.2)$ & $56.5(49.5,93.1)$ & $0.032^{*}$ \\
\hline Creatine kinase (U/L) & $51.2(37.0,73.2)$ & $73.8(54.7,100.9)$ & $52.1(38.4,72.6)$ & $46.7(33.2,72.7)$ & $57.4(29.4,152.4)$ & $0.083^{*}$ \\
\hline $\begin{array}{l}\text { Creatine kinase-MB } \\
(\mathrm{IU} / \mathrm{L})\end{array}$ & $8.6(6.9,10.9)$ & $8.6(7.0,10.4)$ & $8.4(6.9,10.5)$ & $8.9(7.1,11.5)$ & $14.7(12.1,22.7)$ & $0.002^{*}$ \\
\hline Blood glucose (mmol/L) & $4.9(4.5,5.7)$ & $4.7(4.2,4.9)$ & $4.8(4.4,5.5)$ & $5.1(4.5,6.0)$ & $6.4(5.2,8.4)$ & $<0.001^{* *}$ \\
\hline Urea nitrogen (mmol/L) & $4.4(3.6,5.4)$ & $3.7(3.1,4.9)$ & $4.2(3.6,5.2)$ & $4.6(3.7,6.0)$ & $7.5(5.2,10.3)$ & $<0.001^{* *}$ \\
\hline Cystatin C (mg/L) & $0.9(0.8,1.1)$ & $0.8(0.7,1.0)$ & $0.9(0.8,1.0)$ & $1.0(0.9,1.1)$ & $1.2(0.9,1.7)$ & $<0.001^{* *}$ \\
\hline Platelets count $\left(10^{9} / \mathrm{L}\right)$ & $220.0(180.0,271.0)$ & $235.0(202.0,274.0)$ & $220.0(184.0,271.0)$ & $220.0(170.0,267.0)$ & $195.0(89.0,290.0)$ & $0.009^{*}$ \\
\hline $\begin{array}{l}\text { Alanine aminotrans- } \\
\text { ferase (IU/L) }\end{array}$ & $21.7(14.2,36.1)$ & $14.9(10.8,35.3)$ & $22.1(14.3,36.4)$ & $21.3(14.2,35.0)$ & $38.8(16.1,51.5)$ & 0.647 \\
\hline $\begin{array}{l}\text { Aspartate aminotrans- } \\
\text { ferase (IU/L) }\end{array}$ & $19.5(15.4,26.1)$ & $17.9(13.5,25.3)$ & $19.1(15.2,25.3)$ & $20.1(15.7,28.6)$ & $31.2(24.2,48.4)$ & $0.001^{*}$ \\
\hline \multicolumn{7}{|l|}{ Treatment-no. (\%) } \\
\hline Intravenous antibiotics & $847(34.8)$ & $2(8.0)$ & $515(29.7)$ & $294(46.3)$ & $36(90.0)$ & $<0.001^{* *}$ \\
\hline Antivirus treatment & $1233(50.7)$ & $11(44.0)$ & $809(46.7)$ & $392(61.7)$ & $21(52.5)$ & $<0.001^{* *}$ \\
\hline $\begin{array}{l}\text { Traditional Chinese } \\
\text { medicine }\end{array}$ & $2348(96.5)$ & $24(96.0)$ & $1679(96.9)$ & $611(96.2)$ & $34(85.0)$ & $0.007^{*}$ \\
\hline Systemic glucocorticoids & $424(17.4)$ & $3(12.0)$ & $202(11.7)$ & $193(30.4)$ & $26(65.0)$ & $<0.001^{* *}$ \\
\hline $\begin{array}{l}\text { Intravenous immuno- } \\
\text { globin }\end{array}$ & $119(4.9)$ & $0(0.0)$ & $39(2.3)$ & $65(10.2)$ & $15(37.5)$ & $<0.001^{* *}$ \\
\hline $\begin{array}{l}\text { Invasive mechanical } \\
\text { ventilation }\end{array}$ & $42(1.7)$ & $0(0.0)$ & $9(0.5)$ & $16(2.5)$ & $17(42.5)$ & $<0.001^{* *}$ \\
\hline $\begin{array}{l}\text { Noninvasive mechanical } \\
\text { ventilation }\end{array}$ & $68(2.8)$ & $0(0.0)$ & $15(0.9)$ & $27(4.3)$ & $26(65.0)$ & $<0.001^{* *}$ \\
\hline $\begin{array}{l}\text { Extracorporeal mem- } \\
\text { brane oxygenation }\end{array}$ & $3(0.1)$ & $0(0.0)$ & $1(0.1)$ & $0(0.0)$ & $2(5.0)$ & $0.001^{*}$ \\
\hline $\begin{array}{l}\text { Continuous renal- } \\
\text { replacement therapy }\end{array}$ & $22(0.9)$ & $0(0.0)$ & $6(0.3)$ & $6(0.9)$ & $10(25.0)$ & $<0.001^{* *}$ \\
\hline $\begin{array}{l}\text { Convalescence plasma } \\
\text { therapy }\end{array}$ & $111(4.6)$ & $0(0.0)$ & $55(3.2)$ & $51(8.0)$ & $5(12.5)$ & $<0.001^{* *}$ \\
\hline \multicolumn{7}{|l|}{$\begin{array}{l}\text { Clinical outcomes-no. } \\
(\%)\end{array}$} \\
\hline Discharged & 2383 (97.9) & 25 (100.0) & 1724 (99.5) & 614 (96.7) & $20(50.0)$ & $<0.001^{* *}$ \\
\hline Deceased & $50(2.1)$ & $0(0.0)$ & $9(0.5)$ & $21(3.3)$ & $20(50.0)$ & $<0.001^{* *}$ \\
\hline
\end{tabular}

Compared with the clinical classification at admission, the ${ }^{*} P$ value is between 0.05 and 0.001 ; the ${ }^{* *} p$ value $<0.001$

\section{Contributing factors to disease progression and COVID-19 fatality}

Of 1,758 mild and moderate patients at admission, $474(27.0 \%)$ progressed to severe or critical severity during hospitalization. Multi-variable cause-specific Cox proportional hazard model (Table 2) identified that patients with age $60-74$ years $(\mathrm{HR}=1.26,95 \% \mathrm{CI}$ $1.02-1.56),>74$ years $(1.44,1.02-2.03)$, respiratory rate $>20$ times/min $(1.28,1.05-1.57)$, fever (temperature $\left.\geq 37.5{ }^{\circ} \mathrm{C}\right)(1.93,1.21-3.08)$, chest tightness $(1.47$, $1.12-1.92)$, blood glucose $>6.1 \mathrm{mmol} / \mathrm{L}(1.58,1.25-$ $1.98)$, c-reaction protein $>4 \mathrm{mg} / \mathrm{L}(1.45,1.12-1.87)$, lactate dehydrogenase $>250 \mathrm{IU} / \mathrm{L}(1.63,1.20-2.20)$, direct bilirubin $>8 \mu \mathrm{mol} / \mathrm{L}(1.51,1.03,2.21)$, albumin $<40 \mathrm{~g} / \mathrm{L}$ $(1.38,1.07-1.77)$ and lymphocyte count $<1.1^{*} 10^{9} / \mathrm{L}(1.44$, 1.15-1.81) were risk factors for disease progression to 


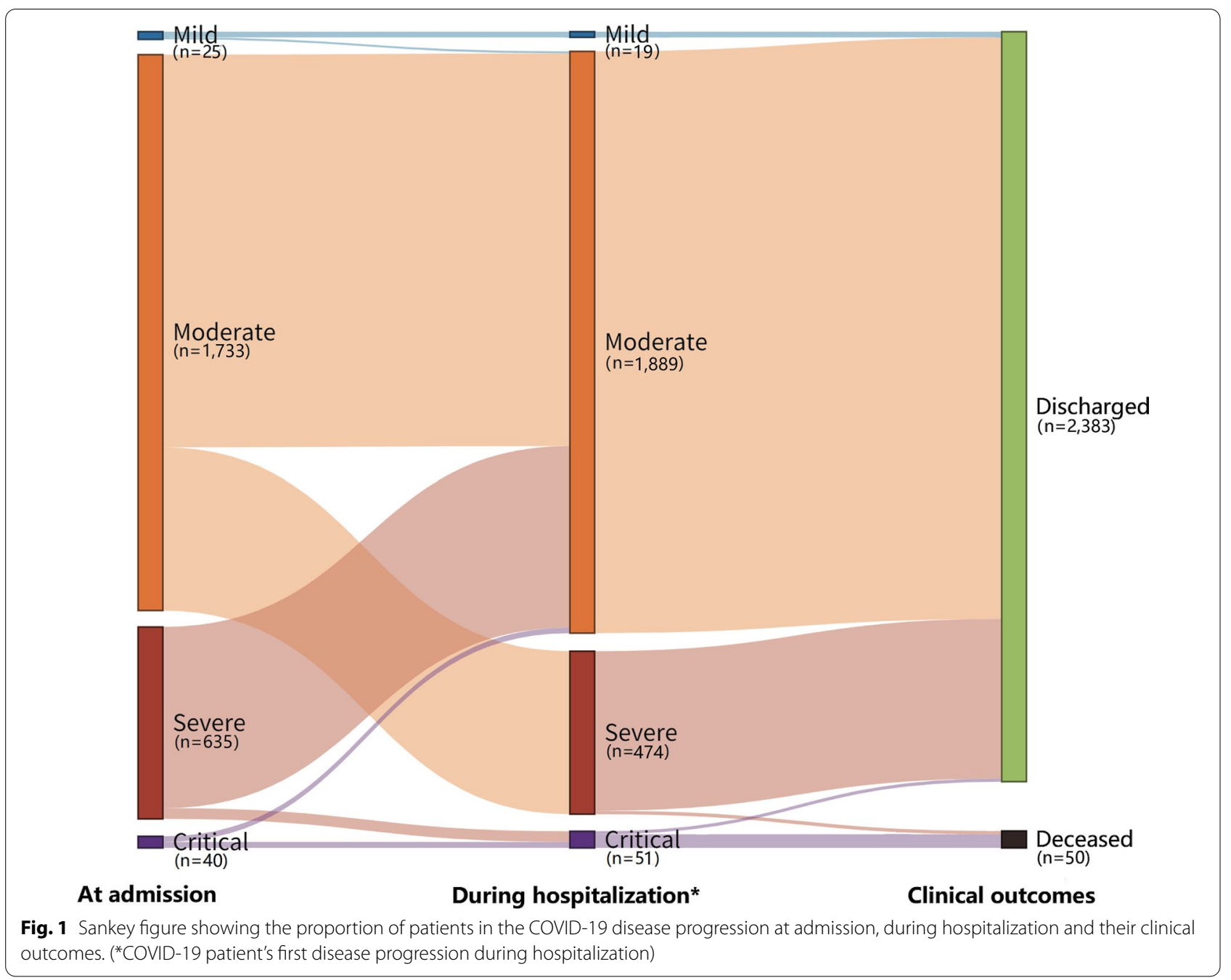

severe and critical stage (Table 2). The 21-day cumulative incidence of progression was $47.8 \%$ in $>74$-years age group, followed by $32.4 \%, 19.8 \%$ in age groups of $60-74$ and $<60$-years respectively. The cumulative incidence of disease progression at day 21 was also much higher in patients with blood glucose $\geq 6.1 \mathrm{mmol} / \mathrm{L}(40.8 \%)$ than blood glucose in range of 3.9-6.1 mmol/L (21.4\%, Fig. 3a, b).

Of 675 severe or critical patients at admission, 634 (93.9\%) were discharged and 41 (6.1\%) died during hospitalization. Table 2 showed that patients with age $>74$ years (3.41, 1.07-10.89), blood glucose $>6.1 \mathrm{mmol} / \mathrm{L}$ (3.22, $1.54-6.73)$, platelets count $<125^{*} 10^{9} / \mathrm{L}(4.39,2.02-9.54)$, fibrinogen $<2 \mathrm{~g} / \mathrm{L}(6.48,1.46-28.67)$ and creatine kinase$\mathrm{MB}>24 \mathrm{IU} / \mathrm{L}(6.29,2.51-15.80)$ were risk factors for in-hospital fatality (Table 2). The 21-day cumulative incidence of fatality was $13.1 \%$ in $>74$ years age group, $4.6 \%$, $1.4 \%$ in age groups of $60-74,<60$ years respectively. The incidence of fatality at day 21 was four times higher in patients with blood glucose $>6.1 \mathrm{~g} / \mathrm{L}(11.8 \%)$ than blood glucose in range of $3.9-6.1 \mathrm{mmol} / \mathrm{L}(2.7 \%$, Fig. $3 \mathrm{c}, \mathrm{d})$.

\section{Discussion}

Our study provides unique progression and outcome data on a cohort of 2,433 COVID-19 patients admitted to Huoshenshan hospital, a hospital designed and built solely to provide care to patients with COVID-19. Our findings suggest that even among inpatients with moderately severe disease, the fatality was relatively low. Most deaths arose from patients who were critically ill on admission or progressed to being critical during admission. The risk factors we identified for death and disease progression are similar to previous studies with older age, poor systematic immune and inflammatory responses, and multiple organ damages [14-16, 18, 24-32]. Patients 


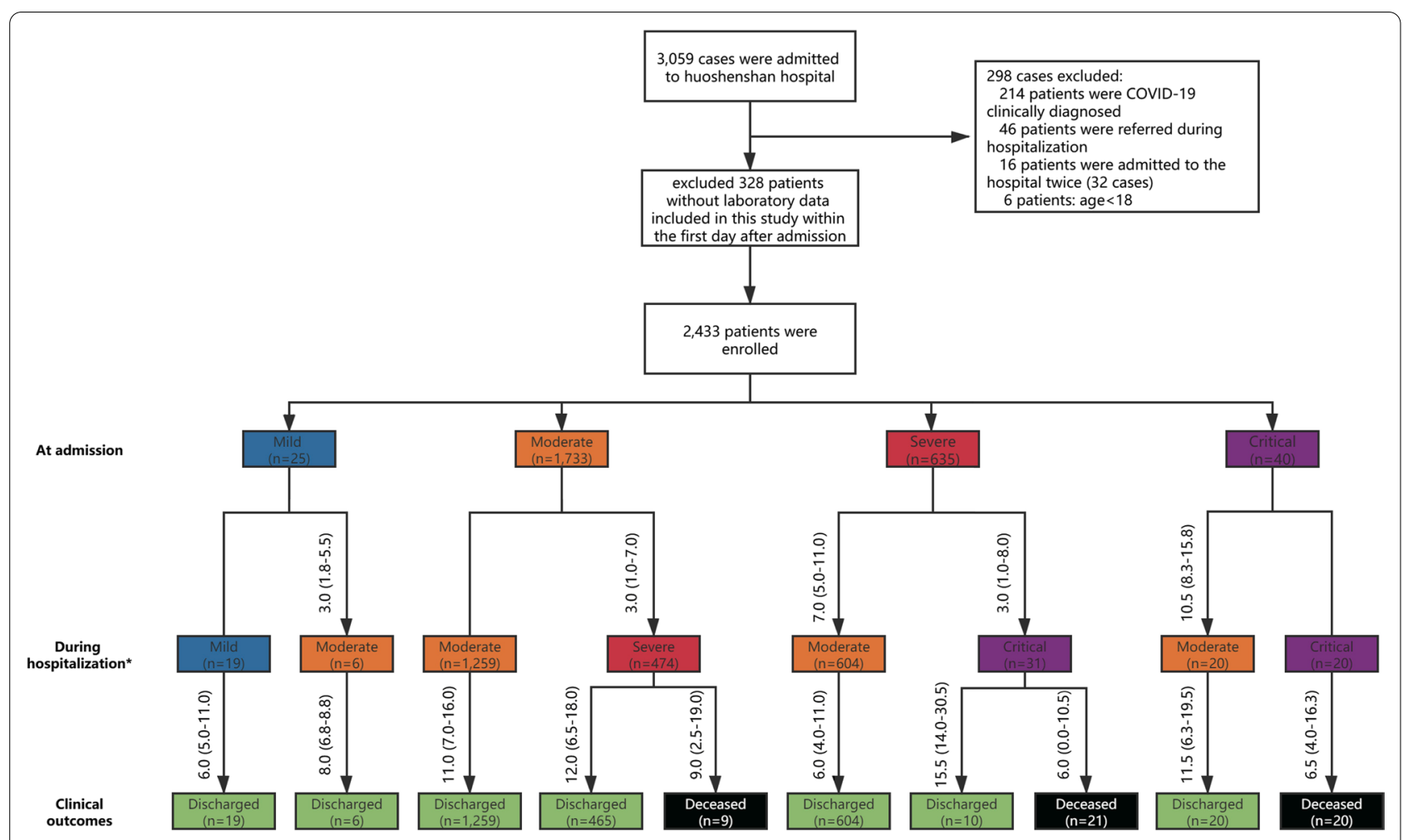

Fig. 2 A flowchart showing the duration in days for disease progression and time to clinical endpoints in 2,433 COVID-19 patients at the Huoshenshan hospital. (*COVID-19 patient's first disease progression during hospitalization)

admitted with a greater disease severity requires longer to recover.

Our report on the time for disease progression at each disease stages allows early preparation and intervention to delay disease progression (Fig. 2). Since there is no effective cure for COVID-19, delaying the progression of the disease is key for survival. Most mild or moderate patients $(73.0 \%)$ did not progress to severe or critical states and recovered from COVID-19 infection in 10.0 (7.0-15.0) days. But among those who did progress, they progressed to a severe or critical stage within the first 3.0 (1.0-7.0) days after admission. This highlights the importance of close monitoring of key risk indicators for disease progression in the early stages of infection. Because, once a patient reaches the critical stage, the chance of fatality is $62.5 \%$ in a short period of $6.5(2.0-13.3)$ days.

We identified the blood glucose level to be an important prognostic predictor for both COVID-19 progression and fatality. In particular, patients with an elevated level of blood glucose $>6.1 \mathrm{mmol} / \mathrm{L}$ had a $58 \%$ higher risk of disease progression and 3.22-fold higher risk of fatality. This is consistent with previous reports that a high glucose level contributes to the development of acute respiratory distress syndrome in COVID-19 patients [16, 17, 28, 29]. Uncontrolled blood glucose level also substantially contributes to other comorbidities, including atherosclerosis, diabetic nephropathy, peripheral arteriosclerosis, and diabetic ketoacidosis, which are also causes of COVID-19 related fatality [28, 33]. The underlying mechanism of how blood glucose interacts with SARSCOV-2 is currently unclear. SARS-COV-2 relies on the binding to membrane angiotensin-converting enzyme-2 (ACE-2) receptor to enter pulmonary cells in the lungs. We speculate that diabetic patients have elevated expression of angiotensin-converting enzyme-2 (ACE-2) receptors, making them vulnerable to SARS-COV-2 infection. Besides, patients living with diabetes or uncontrolled glucose level are likely to have impaired innate immunity due to dysfunction of macrophage and lymphocytes, which may lead to an increased risk of septic shock and multiple organ failures. Hyperglycemia is also a potential indicator of pancreatic islet cell injury [33]. Effective glycemic monitor and management may be beneficial to reduce the progression and fatality of COVID-19 patients $[34,35]$. 
Table 2 Multivariate cox proportional hazards regression for disease progression and fatality among COVID-19 patients in Huoshenshan hospital

\begin{tabular}{|c|c|c|c|c|}
\hline \multirow[t]{2}{*}{ Variable } & \multicolumn{2}{|c|}{$\begin{array}{l}\text { The first disease progression from mild/ } \\
\text { moderate at admission to severe/critical } \\
\text { during hospitalization }\end{array}$} & \multicolumn{2}{|c|}{$\begin{array}{l}\text { Disease fatality among patients } \\
\text { with severe/critical at admission } \\
\text { during hospitalization }\end{array}$} \\
\hline & $\mathrm{HR}(95 \% \mathrm{Cl})$ & $p$ value & HR (95\% Cl) & $P$ value \\
\hline \multicolumn{5}{|l|}{ Demographic characteristics } \\
\hline \multicolumn{5}{|l|}{ Age (year) } \\
\hline$<60$ & Reference & & Reference & \\
\hline $60-74$ & $1.26(1.02,1.56)$ & $0.033^{*}$ & $1.46(0.47,4.58)$ & 0.511 \\
\hline$>74$ & $1.44(1.02,2.03)$ & $0.037^{*}$ & $3.41(1.07,10.89)$ & $0.038^{*}$ \\
\hline Respiratory rate > 20 (\%) & $1.28(1.05,1.57)$ & $0.015^{*}$ & & \\
\hline \multicolumn{5}{|l|}{ Signs and symptoms-no. (\%) } \\
\hline Fever (temperature $\geq 37.5^{\circ} \mathrm{C}$ ) & $1.93(1.21,3.08)$ & $0.007^{*}$ & & \\
\hline Chest tightness & $1.47(1.12,1.92)$ & $0.005^{*}$ & & \\
\hline Fatigue & $1.14(0.94,1.37)$ & 0.169 & & \\
\hline \multicolumn{5}{|l|}{ Laboratory findings } \\
\hline \multicolumn{5}{|l|}{ Blood glucose (mmol/L) } \\
\hline $3.9-6.1$ & Reference & & Reference & \\
\hline$<3.9$ & $1.65(0.97,2.81)$ & 0.065 & $7.31(0.00$, inf $)$ & 0.996 \\
\hline$>6.1$ & $1.58(1.25,1.98)$ & $<0.001^{* *}$ & $3.22(1.54,6.73)$ & $0.002^{*}$ \\
\hline \multicolumn{5}{|l|}{ C-reactive protein (mg/L) } \\
\hline$\leq 4$ & Reference & & & \\
\hline$>4$ & $1.45(1.12,1.87)$ & $0.004^{*}$ & & \\
\hline \multicolumn{5}{|l|}{ D-dimer (mg/L) } \\
\hline$\leq 0.55$ & Reference & & & \\
\hline$>0.55$ & $1.27(0.96,1.67)$ & 0.099 & & \\
\hline \multicolumn{5}{|l|}{ Lymphocyte count $\left(10^{9} / \mathrm{L}\right)$} \\
\hline $1.1-3.2$ & reference & & & \\
\hline$<1.1$ & $1.44(1.15,1.81)$ & $0.002^{*}$ & & \\
\hline$>3.2$ & $0.87(0.31,2.40)$ & 0.784 & & \\
\hline \multicolumn{5}{|l|}{ Lactate dehydrogenase (IU/L) } \\
\hline $120-250$ & Reference & & & \\
\hline$<120$ & $1.38(0.78,2.42)$ & 0.267 & & \\
\hline$>250$ & $1.63(1.20,2.20)$ & $<0.001^{* *}$ & & \\
\hline \multicolumn{5}{|l|}{ Direct bilirubin ( $\mu \mathrm{mol} / \mathrm{L})$} \\
\hline$\leq 8$ & Reference & & & \\
\hline$>8$ & $1.51(1.03,2.21)$ & 0.035 & & \\
\hline \multicolumn{5}{|l|}{ Platelets count $\left(10^{9} / \mathrm{L}\right)$} \\
\hline $125-350$ & Reference & & Reference & \\
\hline$<125$ & $0.65(0.41,1.05)$ & 0.078 & $4.39(2.02,9.54)$ & $<0.001^{* *}$ \\
\hline$>350$ & $1.12(0.82,1.53)$ & 0.480 & $2.60(0.56,11.92)$ & 0.220 \\
\hline \multicolumn{5}{|l|}{ Fibrinogen (g/L) } \\
\hline $2-4$ & Reference & & Reference & \\
\hline$<2$ & $1.16(0.53,2.53)$ & 0.718 & $6.48(1.46,28.67)$ & $0.016^{*}$ \\
\hline$>4$ & $1.19(0.89,1.59)$ & 0.799 & $0.77(0.29,2.09)$ & 0.611 \\
\hline \multicolumn{5}{|l|}{ Monocyte count $\left(10^{9} / \mathrm{L}\right)$} \\
\hline $0.1-0.6$ & Reference & & & \\
\hline$<0.1$ & $0.32(0.07,1.37)$ & 0.125 & & \\
\hline$>0.6$ & $0.96(0.72,1.27)$ & 0.762 & & \\
\hline \multicolumn{5}{|l|}{ Albumin (g/L) } \\
\hline $40-55$ & Reference & & & \\
\hline
\end{tabular}


Table 2 (continued)

\begin{tabular}{|c|c|c|c|c|}
\hline \multirow[t]{2}{*}{ Variable } & \multicolumn{2}{|c|}{$\begin{array}{l}\text { The first disease progression from mild/ } \\
\text { moderate at admission to severe/critical } \\
\text { during hospitalization }\end{array}$} & \multicolumn{2}{|c|}{$\begin{array}{l}\text { Disease fatality among patients } \\
\text { with severe/critical at admission } \\
\text { during hospitalization }\end{array}$} \\
\hline & $\mathrm{HR}(95 \% \mathrm{Cl})$ & $p$ value & HR $(95 \% \mathrm{Cl})$ & $P$ value \\
\hline$<40$ & $1.38(1.07,1.77)$ & $0.013^{*}$ & & \\
\hline$>55$ & 0.0009 (0.00, inf $)$ & 0.992 & & \\
\hline \multicolumn{5}{|c|}{ Aspartate aminotransferase (IU/L) } \\
\hline$\leq 40$ & Reference & & & \\
\hline$>40$ & $1.24(0.90,1.72)$ & 0.189 & & \\
\hline \multicolumn{5}{|c|}{ Neutrophils count $\left(10^{9} / \mathrm{L}\right)$} \\
\hline $1.8-6.3$ & Reference & & & \\
\hline$<1.8$ & $1.24(0.81,1.88)$ & 0.323 & & \\
\hline$>6.3$ & $1.22(0.87,1.71)$ & 0.244 & & \\
\hline \multicolumn{5}{|c|}{ Urea nitrogen (mmol/L) } \\
\hline $2.9-7.5$ & Reference & & & \\
\hline$<2.9$ & $0.83(0.59,1.17)$ & 0.277 & & \\
\hline$>7.5$ & $0.99(0.64,1.53)$ & 0.964 & & \\
\hline \multicolumn{5}{|c|}{ Cystatin C (mg/L) } \\
\hline $0.51-1.09$ & Reference & & & \\
\hline$<0.51$ & $1.37(0.28,7.00)$ & 0.696 & & \\
\hline$>1.09$ & $1.01(0.77,1.31)$ & 0.980 & & \\
\hline \multicolumn{5}{|c|}{ Creatine kinase-MB (IU/L) } \\
\hline$\leq 24$ & & & Reference & \\
\hline$>24$ & & & $6.29(2.51,15.80)$ & $<0.001^{* *}$ \\
\hline
\end{tabular}

Our study reported important clinical indicators that are associated with COVID-19 disease progression and fatality. At the systemic level, a low lymphocyte count is an indication of a poor systematic immune response against the infection, whereas a high c-reactive protein level is an early indicator of systemic inflammatory response syndrome [36]. Besides, increased fibrinogen and platelets count concentrations are associated with increased coagulation activity in patients with infection or sepsis [37, 38]. This is particularly life-threatening for patients with pre-existing cerebro-or cardiovascular diseases, which is common among the elderly. In addition, an elevated creatine kinase-MB level, a know indicator for acute myocardial injury. Further, liver damage or dysfunction, marked by reduced albumin level and elevated direct bilirubin [39]. Most of the remaining indicators may be associated with multiple organ injury or failure. Lactate dehydrogenase predicts the severity of tissue damage. As lactate dehydrogenase is largely present in lung tissues, when damaged by SARS-COV-2 infection, a large amount of lactate dehydrogenase into the circulation. This often clinically presents as a severe form of interstitial pneumonia and subsequently evolve into acute respiratory distress syndrome. Elevated lactate dehydrogenase level is also a predictor of endothelial damage, which caused microvascular thrombosis and associated with renal failure [40]. Maintaining an effective systemic immune response against the infection and prevention of multiple organ failures are, therefore, the priorities in treating COVID-19 patients.

Our study has several limitations. First, this is a retrospective single-centre study, and almost half of patients did not have laboratory tests for interleukin-6, natriuretic peptide type $B$, supersensitive troponin I, myoglobin and procalcitonin levels within the first day after admission. Their role may be underestimated in the prediction of disease progression and fatality. Second, most patients on admission had moderate severity which may lead to a selection bias when identifying factors that affect progression or fatality. Additional cohort studies of patients 


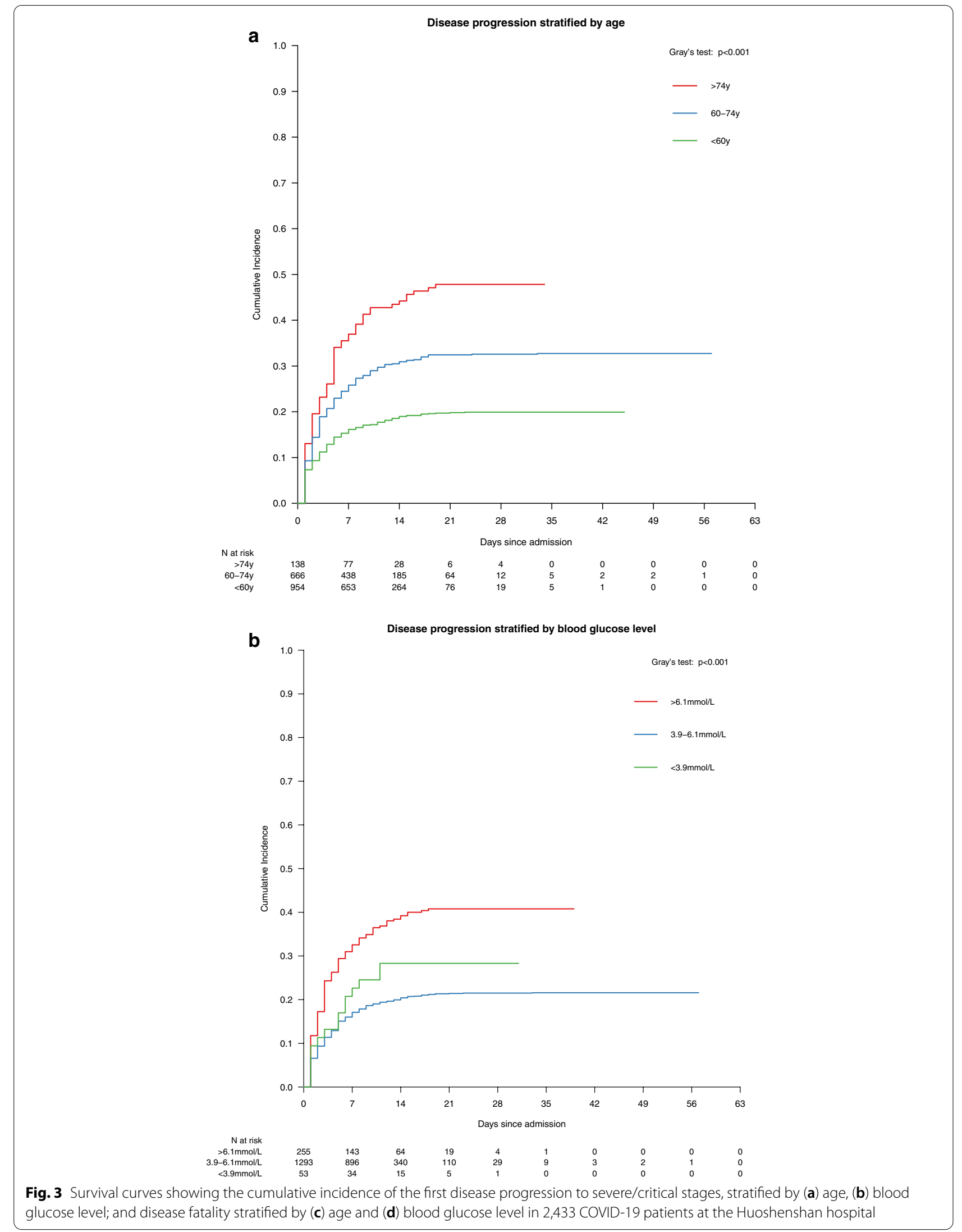




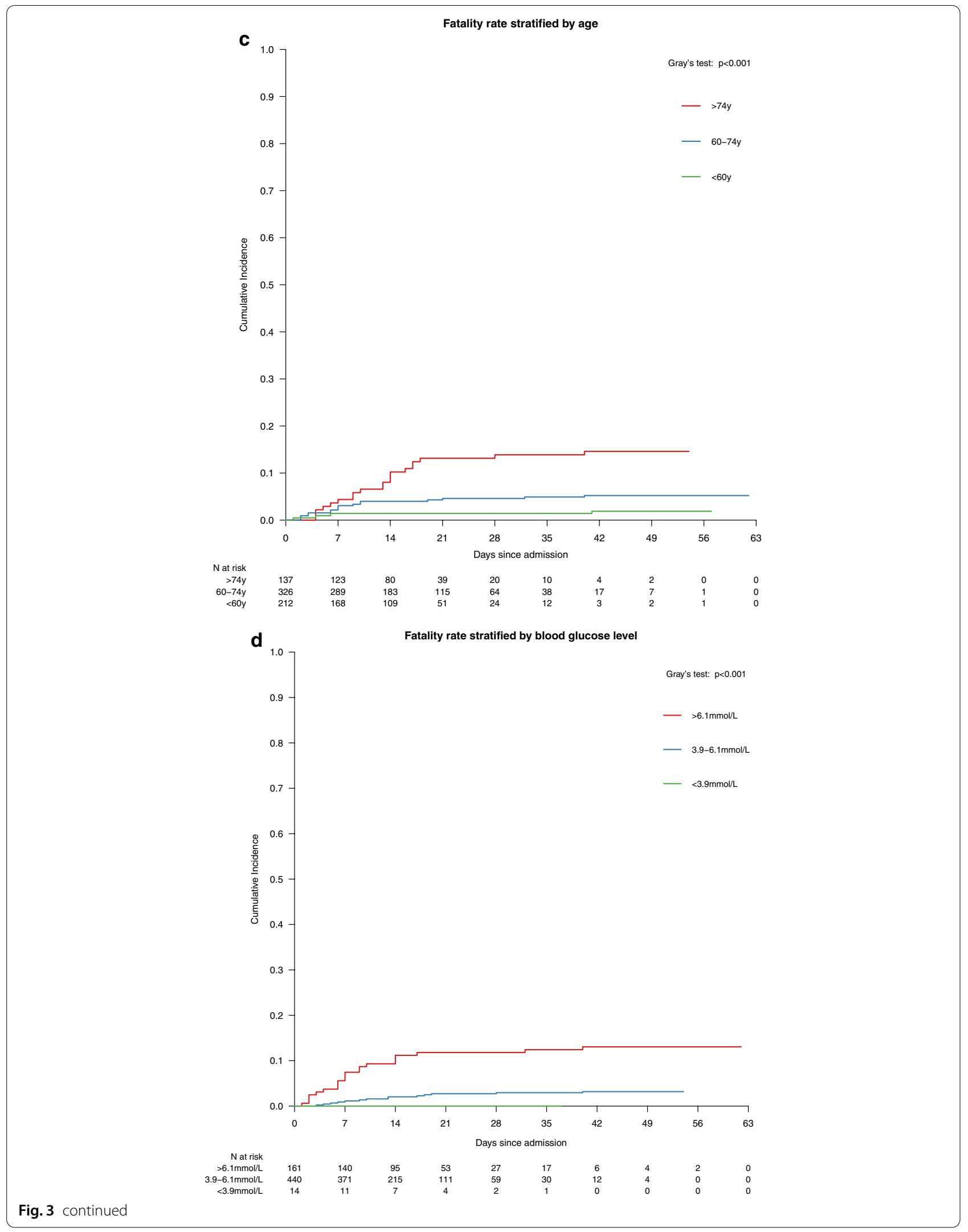


with COVID-19 pneumonia from areas outside Wuhan are needed to confirm our results. Third, considering both the small number of events and the rule of thumb on event per variable $>10$, only limited risk factors were included in the multivariable analysis of severe and critically illness at admission to fatality during hospitalization. Fourth, most patients were referred from other medical institution, and the data at diagnosis was not accessible to us.

\section{Conclusions}

In this study, we comprehensively presented stage-wise disease's first progression among COVID-19 patients. We identified that older age, elevated glucose level, together with other clinical indicators associated with systemic responses and multiple organ failures, predicted both the disease progression and fatality among COVID19 patients.

\section{Supplementary Information}

The online version contains supplementary material available at https://doi. org/10.1186/s12890-021-01413-w.

Additional file 1. Statistical analysis for key indicators of COVID-19 progression, fatality, and associated factors.

\section{Acknowledgements}

We thank all medical staff, researchers and patients who participated in this study. In addition, we especially thank Beijing BioMind Technology company, Mrs. Peifang Zhang, Mr. Zhenzhou Wu, Mr. Baoxin Liao and Mr. Yu Liu for participating.

\section{Authors' contributions}

Participants of the study should "contribute" as follows: (data collection, data analysis, writing). WW: Study design, Data collection, Data analysis, Writing. MS: Writing. YT: Table and figure organization. CKF: Writing. QZ: Data collection. ZL: Data collection. HC: Data collection. JJO: Data collection. DZ: Data collection. KZ: Data collection. NX: Data collection. HG: Data collection. EQ: Data collection. XG: Data collection. FY: Data collection. SZ: Data collection. LZ: Study design, Data collection, Data analysis, Writing. KH: Study design, Data collection, Data analysis, Writing. All authors read and approved the final manuscript.

\section{Funding}

This work was supported by the Beijing Science and Technology Project (No. Z201100005420022).

\section{Availability of data and materials}

The data that support the findings of this study are available from the Huoshenshan hospital but restrictions apply to the availability of these data, which were used under license for the current study, and so are not publicly available. Data are however available from the authors upon reasonable request and with permission of the Huoshenshan hospital.

\section{Ethics approval and consent to participate}

All methods of this study were carried out in accordance with relevant guidelines and regulations. This study was approved by Ethical Review of Scientific Research Projects of the Medical Ethics Committee of the Chinese PLA General Hospital, and obtained a waiver of one or more elements of informed consent form. The need for informed consent was waived by Medical Ethics Committee of the Chinese PLA General Hospital. The name of the ethics committee: The Medical Ethics Committee of the Chinese PLA General Hospital. The approval committee's reference number: No. S2020-162-01.
Consent for publication

Not applicable.

\section{Competing interests}

All authors declared there were no conflicts of interests.

\section{Author details}

${ }^{1}$ Key Laboratory of Ministry of Industry and Information Technology of Biomedical Engineering and Translational Medicine, Chinese PLA General Hospital, Beijing 100853, People's Republic of China. ${ }^{2}$ Translational Medical Research Center, Chinese PLA General Hospital, Beijing 100853, People's Republic of China. ${ }^{3}$ Medical Artificial Intelligence Research Center, Chinese PLA General Hospital, Beijing 100853, People's Republic of China. ${ }^{4}$ China-Australia Joint Research Center for Infectious Diseases, School of Public Health, Xi'an Jiaotong University Health Science Center, Xi'an, Shanxi 710061, People's Republic of China. ${ }^{5}$ Central Clinical School, Faculty of Medicine, Nursing and Health Sciences, Monash University, Melbourne, VIC, Australia. ${ }^{6}$ Department of Medical Information, Huoshenshan Hospital, Wuhan, Hubei, People's Republic of China. ${ }^{7}$ Department of Medical Information, The 940th Hospital of PLA Joint Logistics Support Force, Lanzhou, People's Republic of China. ${ }^{8}$ Department of Infectious Disease, the Fifth Medical Center, Chinese PLA General Hospital, Beijing 100039, People's Republic of China. ${ }^{9}$ Department of Medical Administration, Chinese PLA General Hospital, Beijing 100853, People's Republic of China. ${ }^{10}$ Department of Radiology, Chinese PLA General Hospital, Beijing 100853, People's Republic of China. ${ }^{11}$ Department of Pulmonary and Critical Care Medicine, Chinese PLA General Hospital, Beijing 100853, People's Republic of China. ${ }^{12}$ Melbourne Sexual Health Centre, Alfred Health, Melbourne, Australia.

Received: 5 November 2020 Accepted: 5 January 2021

Published online: 24 February 2021

\section{References}

1. World Health Organization. Coronavirus disease (COVID-2019) situation reports. https://www.who.int/emergencies/diseases/novel-coronaviru s-2019/situation-reports/. Accessed 3 August.

2. China NHCoTPsRo. The latest situation of the new coronavirus pneumonia epidemic situation. http://www.nhc.gov.cn/xcs/yqtb/list_gzbd.shtml. Accessed 2 August.

3. Zhang $L S M, M a X$, et al. What is required to prevent a second major outbreak of the novel coronavirus COVID-19 upon lifting the metropolitanwide quarantine of Wuhan city, China: a mathematical modelling study. Social Science Electronic Publishing 2020.

4. Sun $Y X$, Koh V, Marimuthu K, et al. Epidemiological and clinical predictors of COVID-19. Clin Infect Dis. 2020;71:786-92.

5. Lian JS, Jin X, Hao SR, et al. Analysis of epidemiological and clinical features in older patients with Corona Virus Disease 2019 (COVID-19) out of Wuhan. Clin Infect Dis. 2020;71:740-7.

6. Cao JL, Tu WJ, Cheng WL, et al. Clinical features and short-term outcomes of 102 patients with corona virus disease 2019 in Wuhan, China. Clin Infect Dis 2020

7. Martinez-Gomez X, Curran A, Campins M, et al. Multidisciplinary, evidence-based consensus guidelines for human papillomavirus (HPV) vaccination in high-risk populations, Spain, 2016. Euro Surveill. 2019;24(7):1700857.

8. Today U. China built a hospital in 10 days to battle coronavirus. https:// www.usatoday.com/story/news/world/2020/02/03/coronavirus-photo s-show-wuhan-huoshenshan-hospital-built-10-days/4643377002/. Accessed 9 May.

9. Yi Z. Huoshenshan Hospital now treating novel coronavirus patients. http://www.chinadaily.com.cn/a/202002/04/WS5e398cefa310128217 274deb.html. Accessed 9 May.

10. Daily A. Wuhan's temporary hospitals close as risk of coronavirus decreases. https://www.archdaily.com/935781/wuhans-temporary-hospi tals-close-as-risk-of coronavirus-decreases. Accessed 9 May.

11. Wynants L, Van Calster B, Bonten MMJ, et al. Prediction models for diagnosis and prognosis of covid-19 infection: systematic review and critical appraisal. BMJ. 2020;369:m1328. 
12. Piccininni $M$, Rohmann J, Foresti $L$, et al. Use of all cause mortality to quantify the consequences of covid-19 in Nembro, Lombardy: descriptive study. BMJ. 2020;369:m1835.

13. Mao B, Liu Y, Chai Y, et al. Assessing risk factors for SARS-CoV-2 infection in patients presenting with symptoms in Shanghai, China: a multicentre observational cohort study. Lancet Digit Health. 2020;2:e323-30.

14. Ji D, Zhang $D, X u$ J, et al. Prediction for Progression Risk in Patients with COVID-19 Pneumonia: the CALL Score. Clin Infect Dis. 2020;71:1393-9.

15. Sahu KK, Siddiqui AD. From Hematologist's desk: The effect of COVID-19 on the blood system. Am J Hematol. 2020;95(8):E213-5.

16. Wu C, Chen X, Cai Y, et al. Risk factors associated with acute respiratory distress syndrome and death in patients with coronavirus disease 2019 pneumonia in Wuhan. China JAMA Intern Med. 2020;180(7):1-11.

17. Zhou F, Yu T, Du RH, et al. Clinical course and risk factors for mortality of adult inpatients with COVID-19 in Wuhan, China: a retrospective cohort study. The Lancet. 2020;395(10229):1054-62.

18. Liang WH, Liang HR, Ou LM, et al. Development and validation of a clinical risk score to predict the occurrence of critical illness in hospitalized patients with COVID-19. JAMA Intern Med. 2020;180:1081-9.

19. Bellou V TI, Evangelou $E$, et al. Risk factors for adverse clinical outcomes in patients with COVID-19: a systematic review and meta-analysis. medRxvi [preprint] 2020

20. Diagnosis and Treatment Protocol for Novel Coronavirus Pneumonia (Trial Version 7). Chinese Medical Journal 2020; 133(9):1087-95.

21. ICD-10-CM official coding and reporting guidelines. 2020

22. Simon NFJ, Hastie T, Tibshirani R. Regularization paths for Cox's proportional hazards model via coordinate descent. J Stat Softw. 2011;39(5):1-13.

23. Peduzzi PCJ, Kemper E, Holford TR, Feinstein AR. A simulation study of the number of events per variable in logistic regression analysis. J Clin Epidemiol. 1996:49(12):1373-9.

24. Jewell NP, Lewnard JA, Jewell BL, et al. Predictive Mathematical Models Of The COVID-19 pandemic: underlying principles and value of projections. JAMA. 2020;323(19):1893-4

25. Guan WJNZ, Hu Y, et al. Clinical characteristics of coronavirus disease 2019 in China. N Engl J Med. 2020;382:1708-20.

26. Wang D, Hu B, Hu C, et al. Clinical characteristics of 138 hospitalized patients with 2019 novel coronavirus-infected pneumonia in Wuhan. China JAMA. 2020;323(11):1061-9.

27. Young BE, Ong SWX, Kalimuddin S, et al. Epidemiologic features and clinical course of patients infected with SARS-CoV-2 in Singapore. JAMA. 2020:323(15):1488-94.
28. Cummings MJBM, Abrams D. Epidemiology, clinical course, and outcomes of critically ill adults with COVID-19 in New York City: a prospective cohort study. Lancet. 2020;395(10239):1763-70.

29. Grasselli G, Zanella A. Critically ill patients with COVID-19 in New York City. The Lancet. 2020;395:1740-1.

30. Paranjpe I, Fuster V, Lala A, et al. Association of treatment dose anticoagulation with in-hospital survival among hospitalized patients with COVID19. J Am Coll Cardiol. 2020;76(1):122-4.

31. Wang S, Zhou X, Zhang T, Wang Z. The need for urogenital tract monitoring in COVID-19. Nat Rev Urol. 2020;17:314-5.

32. Qi XL, Liu Y, Wang JT, et al. Clinical course and risk factors for mortality of COVID-19 patients with pre-existing cirrhosis: a multicentre cohort study. Gut. 2021;70:433-6.

33. Bode BGV, Messler J, et al. Glycemic characteristics and clinical outcomes of COVID-19 patients hospitalized in the United States. J Diabetes Sci Technol. 2020;14(4):813-21.

34. Sinclair AJ, Abdelhafix AH. Age, frailty and diabetes - triple jeopardy for vulnerability to COVID-19 infection. EClinicalMedicine. 2020;22:100343.

35. Ceriello A. Hyperglycemia and the worse prognosis of COVID-19. Why a fast blood glucose control should be mandatory. Diabetes Res Clin Pract. 2020;163:108186.

36. Sierra R, Rello J, Bailen MA, et al. C-reactive protein used as an early indicator of infection in patients with systemic inflammatory response syndrome. Intensive Care Med. 2004;30(11):2038-45.

37. Zhang Y, Xiao M, Zhang SL, et al. Coagulopathy and antiphospholipid antibodies in patients with Covid-19. N Engl J Med. 2020;382:e38.

38. Milbrandt EB, Reade MC, Lee $M$, et al. Prevalence and significance of coagulation abnormalities in community-acquired pneumonia. Mol Med. 2009;15(11-12):438-45.

39. Wang SH HP, Xiao F. Manifestations of liver injury in 333 hospitalized patients with coronavirus disease 2019. Chin J Dig 2020.

40. Wang X, Sahu KK, Cerny J. Coagulopathy, endothelial dysfunction, thrombotic microangiopathy and complement activation: potential role of complement system inhibition in COVID-19. J Thromb Thrombolysis. 2020:1-6.

\section{Publisher's Note}

Springer Nature remains neutral with regard to jurisdictional claims in published maps and institutional affiliations.
Ready to submit your research? Choose BMC and benefit from:

- fast, convenient online submission

- thorough peer review by experienced researchers in your field

- rapid publication on acceptance

- support for research data, including large and complex data types

- gold Open Access which fosters wider collaboration and increased citations

- maximum visibility for your research: over $100 \mathrm{M}$ website views per year

At BMC, research is always in progress.

Learn more biomedcentral.com/submissions 